\title{
Comparison of immunofluorescence assay (IF) with ELISA in detection of antinuclear antibodies
}

\author{
Ashish Tayde ${ }^{1}$, Chetna Agrawal, ${ }^{2, *}$ A. T. Deshmukh ${ }^{3}$ \\ ${ }^{\mathbf{1}}$ Associate Professor, ${ }^{\mathbf{2}}$ Assistant Professor, ${ }^{\mathbf{3}}$ Professor and Head, Dept. of Pathology, Dr. Panjabrao Deshmukh Memorial Medical \\ College, Amravati, Maharashtra, India \\ *Corresponding Author: \\ Email: drchetnaagrawal@gmail.com
}

Received: $18^{\text {th }}$ December, 2017

Accepted: $23^{\text {rd }}$ February, 2018

\begin{abstract}
Patients with autoimmune connective tissue diseases often have antibodies that are directed against multiple nuclear antigens called as antinuclear antibodies (ANA). Two testing methods i.e. ELISA and indirect immunofluorescence (IF) techniques are used to detect these antibodies. Though ELISA is a cheaper method, IF is a preferred method for detection of ANA. In our study we have compared these two techniques for their diagnostic performance. Both the testing methods were applied on 155 samples. Of these, 135 samples were from test group and 20 samples were controls (negative and positive controls). Of the 135 test samples, IF yield positive results in $25(18.51 \%$ ) cases and was found negative in $110(81.49 \%)$ cases. Positive results were found by ELISA in $20(14.81 \%)$ cases and negative in $115(85.19 \%)$ cases. Samples showing positive results with both methods were $18(13.33 \%)$ and samples showing negative results with both methods were 108(80.0\%). 07(5.18\%) cases that gave negative results by ELISA were found to be positive by IF. 02(1.48\%) samples that were found to be positive by ELISA were negative by IF. Sensitivity \& specificity of ELISA was compared with IF and was found to be $90.0 \%$ and $93.9 \%$ respectively. From this study it can be concluded that for testing ANA, IF is better than ELISA.
\end{abstract}

Keywords: IF (Immunofluorescence), ELISA (Enzyme Linked Immunosorbent Assay), ANA (Antinuclear Antibodies).

\section{Introduction}

Patients having autoimmune connective tissue disease often have antibodies that are directed against multiple nuclear antigens called as antinuclear antibodies (ANA). ${ }^{1}$ These antibodies are also the basis for diagnosis and treatment of these diseases. ${ }^{2}$ In connective diseases like SLE, CREST syndrome, Sjogren's syndrome, scleroderma, mixed connective disorders etc., ANA testing is widely used as screening test. ${ }^{3}$ Two testing methods which are used in detection of ANA are ELISA (Enzyme Linked Immunosorbent Assay) and indirect immunofluorescence (IF), but the preferred technique is indirect immunofluorescence. ${ }^{4}$ In detecting ANA by indirect immunofluorescence method various nuclear staining patters are observed that include homogenous, speckled, membranous, centromeric, pleomorphic etc. The staining pattern of antibodies and intensity allows skilled observer to distinguish between numerous antinuclear antibodies. ${ }^{5}$ Certain ANA IF patterns are associated with the presence of autoantibodies to certain nuclear antigens which in turn are associated with certain clinical state. ${ }^{6}$ However, the results by IF method is affected by fixation procedure, inspection time, dilution of serum, expertise of technician and also microscope itself. ${ }^{7}$ To overcome these influencing factors of ANA by IF, ANA by ELISA can be used as attractive alternative with added advantage of speed and simplicity of the test. Even some ELISA assays approach Immunofluorescence in their sensitivity and specificity. ${ }^{8,9}$ Despite having advantage of speed and simplicity most ANA testing by ELISA have a disadvantage due to reduced antigen diversity leading to decrease sensitivity.

The present study was done to detect ANA by IF and ELISA method in patients with clinical symptoms of autoimmune connective tissue diseases. Results of both the tests were then compared to see the diagnostic performances.

\section{Materials and Methods}

This was a prospective study conducted in Research laboratory, Dept. of Pathology, Dr. Panjabrao Deshmukh Memorial Medical College, Amravati over a period of one year from November 2015 to October 2016. Prior to test informed consent from patients was taken. ANA by IF and ELISA was carried out in 155 blood samples. Out of these samples, 135 samples were taken from patients with clinical symptoms of autoimmune diseases.

Inclusion Criteria for Patient selection: Patients with history and signs \& symptoms suggestive of autoimmune connective tissue disease.

Exclusion Criteria for Patient Selection: Patients having established diagnosis of autoimmune disease and were under treatment. 10 samples were withdrawn from healthy individuals who served as negative control (without any sign and symptoms of disease) and 10 samples taken from patients with known autoimmune disease and were considered as positive control (Already diagnosed autoimmune connective tissue disease patients). ANA by IF was carried out as per 
instructions provided by manufacturer of kit (HEP 2000, immunoconcepts, USA). IF was performed on serum with dilution of 1:80. Diluted sera were placed on ANA wells provided in kit which allowed anti nuclear antibodies present in sera to bind with the corresponding antigens present on the slides. The slides were than incubated for 30 mins and were then rinsed with phosphate buffer. Fluorescein labeled antihuman globulin provided in test kit was added, incubated for 30 more mins, rinsed, followed by mounting with mounting medium and slides were examined under the fluorescent microscope for staining pattern and intensity. When observed under IF microscope varied patterns with different intensity was seen. These patterns were homogenous, speckled, nucleolar and centromere. ANA ELISA was performed on same serum samples as per kit manufacturer's instructions. Sera was diluted and added to the nuclear antigen coated wells provided by the kit manufacturer. Wells were rinsed to provide only bound ANA on the wells. Enzyme conjugate was added to these wells with antigen antibody complexes. Wells were again rinsed to remove excess conjugate and incubated. Intensity of colour is then read on ELISA reader which will be proportional to the $\operatorname{IgG}$ specific antibodies in the sera. The results thus obtained by both IFA techniques were then compared.

\section{Results}

ANA by immunofluorescence (IF) and ELISA was done on 155 samples which includes 135 samples as test group and 20 samples as control group (10 positive control and 10 negative control). Of the test group, $31(22.96 \%)$ were males and $104(77.04 \%)$ were females. Out of 104 females of test group, 24(23.08\%) showed ANA positivity and out of 31 males of test group, 01(3.23\%) showed ANA positivity. Of the 135 test samples of test group positive result by IF was seen in $25(18.51 \%)$ cases and negative in $110(81.49 \%)$ cases. Positive result by ELISA method was seen in $20(14.81 \%)$ samples and negative results were seen in 115(85.19 \%) cases. By both methods 18(13.33\%) samples were tested positive and 108 samples (80\%) tested negative. $07(5.18 \%)$ cases that gave negative results by ELISA were found to be positive by IF. 02(1.48\%) samples that were found to be positive by ELISA were negative by IF (Table $2 \& 3$ ). All controls gave desired results with both methods for ANA detection. Various patters were observed in IF positive cases as shown in table 1.

Table 1: Showing fluorescence pattern observed in
IF positive cases
\begin{tabular}{|l|c|}
\hline $\begin{array}{c}\text { Fluorescence Pattern } \\
\text { observed }\end{array}$ & Number of samples \\
\hline Speckled & $14(56 \%)$ \\
\hline Homogenous & $08(32 \%)$ \\
\hline Nucleolar & $01(4 \%)$ \\
\hline Centromere & $02(8 \%)$ \\
\hline Total & $25(100 \%)$ \\
\hline
\end{tabular}

These patterns showed varying intensity of $1+, 2+$ and $3+$.

Table 2: Showing IF and ELISA results

\begin{tabular}{|l|c|c|c|}
\hline Testing Method & Results & Cases & Percentage \\
\hline Immunofluorescence method & Positive & 25 & $18.51 \%$ \\
\cline { 2 - 4 } & Negative & 110 & $81.49 \%$ \\
\hline Total & Positive & 135 & $100 \%$ \\
\hline \multirow{2}{*}{ ELISA method } & Negative & 115 & $14.81 \%$ \\
\hline \multicolumn{2}{|l|}{ Total } & 135 & $85.19 \%$ \\
\hline
\end{tabular}

Table 3: Comparison of ANA by IF and ELISA methods

\begin{tabular}{|l|c|c|c|}
\hline \multirow{2}{*}{\multicolumn{1}{c|}{ IF }} & \multicolumn{2}{c|}{ ELISA } & \multirow{2}{*}{ Total } \\
\cline { 2 - 3 } & ANA Positive & ANA Negative & 25 \\
\hline ANA Positive & 18 & 07 & 110 \\
\hline ANA Negative & 02 & 108 & 135 \\
\hline Total & 20 & 115 & \\
\hline
\end{tabular}

In this study IF was done on 10 positive controls and 10 negative controls and it was found that all controls gave desired results. Taking this into consideration we compared ELISA method with IF. Sensitivity and specificity of ELISA when compared with IF was calculated to be $90.0 \%$ and $93.9 \%$ respectively. Positive predictive value and negative predictive value of ELISA when compared with IFA was found to be $72 \%$ and $98.18 \%$ respectively.

\section{Discussion}

In this study total 135 cases suspected clinically to have Autoimmune Connective tissue disease were studied out of which 104(77.03\%) were females and $31(22.97 \%)$ were males. This finding correlates with 
study of Priyadarshani et $\mathrm{al}^{10}$ in which 75 cases were studied which included 57(76.0\%) females and $18(24.0 \%)$ males. In present study ANA positivity was more among females [ie. $2423.08 \%$ ) cases out of 104 female suspects] than males [i.e. 01(3.23\%) case in 31 suspects]. This finding correlates with study of Hyashi et al. $^{11}$ and Priyadarshani et $\mathrm{al}^{10}$ This suggests that autoimmune diseases are more common among females.

In this study among 135 test group cases ANA by IF showed positivity in $25(18.52 \%)$ cases and negative in $110(81.48 \%)$ cases while ANA by ELISA showed positivity in $20(14.81 \%)$ cases and negative in $115(85.19 \%)$ cases. ANA positivity by IF was more when compared with ELISA. This can be explained as ELISA was unable to detect low titre of antibodies present in test sera and is also unable to detect all types of antibodies. The above findings correlate with the studies of Priyadarshani et al., ${ }^{10}$ Dipiti et al. $^{2}$ and Fawcett et al. ${ }^{12}$

Among 135 test samples tested for ANA by IF and ELISA, $18(13.33 \%)$ samples were tested positive by both methods and 108 samples $(80 \%)$ tested negative by both methods. $07(5.18 \%)$ cases that gave negative results by ELISA were found to be positive by IF. 02 (1.48\%) samples that were found to be positive by ELISA were negative by IF. HEP2 cells (used in IF) are spindle shaped cells derived from human epithelial cell line of laryngeal carcinoma and since it is of human origin, it replicates all antigens in a human cell and also has a greater yield due to high mitotic rate. In IF more than 20 patterns can be observed and it represents more than 100 antigens. In contrast most ELISA kits are coated with fewer antigens (around 20), both recombinant and human antigen are used resulting in greater likelihood of false negativity. False negativity in ANA by IF is rare; however SSA antigens are under represent in HEP2 slide due to difference in methodology of extraction. SSA antigens are susceptible to acetone extraction and hence degraded easily. ${ }^{13}$

\section{Conclusion}

Significant difference was observed in detection of ANA when done by two techniques i.e. IF and ELISA. This may be due to difference in array of antigen present in test kits. From this study it can be concluded that for testing ANA, IF is better than ELISA. However, it was observed that both methods if done together will increase specificity of ANA testing. IF can be considered as gold standard method in ANA detection and we recommend use IF method for ANA detection.

\section{References}

1. Slater CA, Davis RB, Shmerling RH, 1996. Antinuclear antibody testing. A study of clinical utility. Arch. Intern. Med., 156(13):1421-1425.
2. Tanjeem Rabika Dipti, Mohammad ShaifulAzam, Mohammad Humayun Sattar, Shahana Rahman. Detection of Antinuclear Antibody in Childhood Rheumatic Diseases by Immunofluorescence Assay and Enzyme Immuno Assay. Bangladesh Journal of Child Health, 35 (2):49-52 (2011).

3. Richard A. Gniewek, Daniel P. Stites, Thomas M. MacHugh, Joan F. Hilton, Mayumi Nakagawa. Comparison of antinuclear antibody testing methods: Immunofluorescence assay versus enzyme immunoassay. Clinical and Diagnostic Laboratory Immunology, March 1997, Vol. 4, no. 2, pg 185-188.

4. Prost, AC et al. 1987. Comparing Hep-2 cell line with rat liver in routine screening test for antinuclear and antinucleolar antibodies in autoimmune disease. Ann. Biol. Clin. 45:610-617.

5. G. Servais, R. Karmali, M.P. Guillaume, V. Badot, J. Duchateaub and F. Corazza. Anti DNA antibodies are not restricted to a specific pattern of Fluorescence on Hep-2 cells. Clinical Chemistry and Laboratory Medicine, Vol. 47, No. 5, pp.543-549, 2009.

6. Gniewek RA, Stites D.P., Thomas M. M, Joan FH and Mayumi Nakagawa. Comparison of Antinuclear Antibody Testing Methods: Immunofluorescence Assay versus EnzymeImmunoassay. Clinical and Diagnostic Laboratory Immunology, 4(2):185-188, (1997).

7. B. N. Pham, S. Albarede, A. Guyard, E. Burg, and T. Maisonneuve. Impact of xternal quality assessment on antinuclear antibody detection performance. Lupus, Vol.14, no.2, pp113-119, 2005.

8. Eric L. Greidinger, Robert W. Hoffman, DO Antinuclear Antibody Testing: Methods, Indications, and Interpretation. Laboratory medicine, 34(2), (2003).

9. Susan S. Copple, Allen D. Sawitzke, MD, Andrew M. Wilson, Anne E. Tebo, Harry R. Hill, Enzyme-Linked Immunosorbent Assay Screening then Indirect Immunofluorescence Confirmation of Antinuclear Antibodies - A Statistical Analysis. Am J Clin Pathol, 135:678-684, (2011).

10. Priyadarshini Shanmugam, Jeya Meenakshi Sundaram and Pothini J. A comparative Study of Enzyme Linked Immunosorbent Assay (ELISA) With

Immunofluorescence Assay (IFA) For the Detection of Antinuclear Antibodies. Int J Pharm Bio Sci 2013 Oct;4(4):(B) 75-80.

11. Nobuhide Hayashi, Tomoko Kawamoto, Masahiko Mukai, Akio Morinobu, Masahiro Koshiba et al. Detection of Antinuclear Antibodies by Use of an Enzyme Immunoassay with Nuclear HEp-2 Cell Extract and Recombinant Antigens: Comparison with Immunofluorescence Assay in 307 Patients. Clinical Chemistry, 47 (9):1649-1659, (2001).

12. Fawcett T, Victoria L M, Carlos D R. Comparison of multiplex, ELISA and immunofluorescence for detecting autoantibodies in JIA patients. Pediatric Rheumatology, 10(1):A117:1-2, (2012).

13. J. Euphrasia Latha, Dhason Therese Mary, Kavitha Mohanasundaram and Sankaralingam Rajeswari. Comparison of Performance of ELISA with Indirect Immunofluorescence for the testing of Antinuclear Antibodies. Int. J. Curr. Microbiol. App. Sci (2016) 5(12):423-427.

How to cite this article: Tayde A, Agrawal C, Deshmukh A. T. Comparison of immunofluorescence assay (IF) with ELISA in detection of antinuclear antibodies. Ind J Pathol Oncol, 2018;5(3):418-420. 